Article

\title{
Key Genes of Lipid Metabolism and WNT-Signaling Are Downregulated in Subcutaneous Adipose Tissue with Moderate Weight Loss
}

\author{
Ruth Schübel ${ }^{1}$, Disorn Sookthai ${ }^{1}$, Judith Greimel ${ }^{1}{ }^{\circledR}$, Theron S. Johnson ${ }^{1}$, \\ Mirja E. Grafetstätter ${ }^{1}$, Romy Kirsten ${ }^{2,3}$, Mario Kratz ${ }^{4}$, Cornelia M. Ulrich ${ }^{5}$, \\ Rudolf Kaaks ${ }^{1}$ and Tilman Kühn ${ }^{1, * \mathbb{D}}$ \\ 1 German Cancer Research Center (DKFZ), Division of Cancer Epidemiology, Im Neuenheimer Feld 581, \\ 69120 Heidelberg, Germany; ruth.schuebel@gmx.de (R.S.); disorn.s@gmail.com (D.S.); \\ judithgreimel@gmail.com (J.G.); t.johnson@dkfz.de (T.S.J.); m.grafetstaetter@dkfz.de (M.E.G.); \\ r.kaaks@dkfz.de (R.K.) \\ 2 Biobank of the National Center for Tumor Diseases (NCT) Heidelberg, Im Neuenheimer Feld 460, \\ 69120 Heidelberg, Germany; romy.kirsten@nct-heidelberg.de \\ 3 Institute of Pathology, Heidelberg University Hospital, Im Neuenheimer Feld 224, \\ 69120 Heidelberg, Germany \\ 4 Division of Public Health Sciences, Fred Hutchinson Cancer Research Center, Seattle, WA 98109, USA; \\ mkratz@fredhutch.org \\ 5 Huntsman Cancer Institute and Department of Population Health Sciences, University of Utah, \\ 2000 Circle of Hope, Salt Lake City, UT 84112-5550, USA; neli@hci.utah.edu \\ * Correspondence: t.kuehn@dkfz.de; Tel.: +49-6221-42-3184
}

Received: 23 February 2019; Accepted: 12 March 2019; Published: 16 March 2019

\begin{abstract}
Smaller cross-sectional studies and bariatric surgery trials suggest that weight loss may change the expression of genes in adipose tissue that have been implicated in the development of metabolic diseases, but well-powered intervention trials are lacking. In post hoc analyses of data from a 12-week dietary intervention trial initially designed to compare metabolic effects of intermittent vs. continuous calorie restriction, we analyzed the effects of overall weight loss on the subcutaneous adipose tissue (SAT) transcriptome. Changes in the transcriptome were measured by microarray using SAT samples of 138 overweight or obese individuals (age range: 35-65 years, BMI range: 25-40, non-smokers, non-diabetics). Participants were grouped post hoc according to the degree of their weight loss by quartiles (average weight loss in quartiles 1 to 4 : $0 \%,-3.2 \%,-5.9 \%$, and $-10.7 \%$ ). Candidate genes showing differential expression with weight loss according to microarray analyses were validated by reverse transcription quantitative polymerase chain reaction (RT-qPCR), and fold changes (FCs) were calculated to quantify differences in gene expression. A comparison of individuals in the highest vs. the lowest weight loss quartile revealed 681 genes to be differentially expressed (corrected $p<0.05$ ), with 40 showing FCs of at least 0.4. Out of these, expression changes in secreted frizzled-related protein 2 (SFRP2, FC $=0.65, p=0.006$ ), stearoyl-CoA desaturase $(\mathrm{SCD}, \mathrm{FC}=-1.00$, $p<0.001$ ), and hypoxia inducible lipid droplet-associated (HILPDA, FC $=-0.45, p=0.001$ ) with weight loss were confirmed by RT-qPCR. Dietary weight loss induces significant changes in the expression of genes implicated in lipid metabolism (SCD and HILPDA) and WNT-signaling (SFRP2) in SAT.
\end{abstract}

Keywords: adipose tissue; gene expression; obesity; weight loss; transcriptomics 


\section{Introduction}

Obesity, characterized by excessive accumulation of adipose tissue, is a risk factor for major chronic diseases such as type 2 diabetes, cardiovascular diseases, and many types of cancer [1-3]. Dysfunctional adipose tissue signaling is a hallmark of obesity and drives its comorbidities, e.g., via increased release of inflammatory factors, altered adipokine profiles, and constant anabolic stimuli by growth factors and hormones [3,4].

For the prevention of pathophysiological complications among overweight or obese people, it has been recommended to aim at $5-10 \%$ weight loss by calorie restriction (CR) approaches and/or increase in physical activity levels [5]. Although the central role of adipose tissue accumulation, particularly in the visceral compartment, is undisputed, there is limited knowledge on the reversibility of obesity-induced alterations in adipose tissue function with weight loss. Adipose tissue gene expression has been used to investigate obesity-related metabolic dysfunction in human trials, as it may provide the basis for the understanding of dysfunctional adipose tissue signaling and its reversibility by weight loss [6,7]. However, many previous studies have been cross-sectional studies with inter-individual comparisons of lean and obese individuals [8,9], or bariatric surgery interventions with substantial weight loss $[10,11]$, while not much is known about the effects of diet-induced weight loss on the adipose tissue transcriptome from larger intervention trials. Moreover, only the expression of specific candidate genes was analyzed in many previous studies, while there is a lack of comprehensive transcriptome-wide, i.e., microarray-derived gene expression data from dietary intervention trials (see Table S1 for a systematic overview).

Here, we evaluated the effects of CR-induced weight loss on abdominal subcutaneous adipose tissue (SAT) gene expression in post hoc analyses from the HELENA Trial (Healthy nutrition and energy restriction as cancer prevention strategies: a randomized controlled intervention trial), a dietary intervention trial among 150 overweight and obese individuals. In this trial, participants achieved moderate weight loss of 7.1\% (with intermittent calorie restriction in form of the 5:2 diet), $5.2 \%$ (continuous calorie restriction), and 3.3\% (control group) over 12 weeks [12]. While the main analyses of the trial did not show differences in SAT gene expression across the three study arms, the goal of the present analyses was to evaluate whether the response to the dietary interventions, i.e., the overall degree of weight loss that was achieved by either method, had an impact on SAT gene expression. Furthermore, we analyzed if the protein products of genes differentially expressed with weight loss in SAT are similarly regulated in serum, and if gene expression and protein levels are related to body mass index (BMI) and established clinical markers of lipid and glucose metabolism (high density lipoprotein [HDL]-cholesterol, low density lipoprotein [LDL]-cholesterol, insulin, homeostatic model assessment for insulin resistance [HOMA-IR] values, and C-reactive protein [CRP]).

\section{Materials and Methods}

\subsection{Study Design and Participants}

One hundred fifty overweight or obese (BMI $>25$ and $\leq 40 \mathrm{~kg} / \mathrm{m}^{2}$ ) men and women (age 35 to 65 years) participated in the HELENA Trial, a randomized controlled intervention study (Clinical Trials.gov, NCT02449148). The HELENA Trial was approved by the ethics committee of the medical faculty Heidelberg (University of Heidelberg, Germany) and had the aim to investigate the effect of timing of calorie restriction (intermittent vs. continuous) on metabolic outcomes including adipose tissue gene expression. Details on study design, power-calculation, recruitment procedures, study assessments, and intervention protocol have been published previously [12,13]. Briefly, all participants were non-smokers, did not have severe chronic diseases (diabetes, major cardiovascular diseases, cancer, or hepatic or kidney dysfunction) according to a medical interview and routine blood parameters prior to commencement of the trial, and did not take lipid-lowering medication. By block randomization, participants were assigned to one of the three study arms: intermittent calorie restriction (ICR; 5 days per week at $0 \%$ energy deficit and 2 days at $75 \%$ deficit), continuous 
calorie restriction (CCR; daily energy deficit 20\%), or a control regimen (without energy limitations), after written informed consent had been obtained. All three groups received general recommendations for a healthy and balanced diet according to the official guidelines of the German Society for Nutrition [14]. At baseline and after the controlled intervention phase of 12 weeks, participants attended the study center for the study assessments, including anthropometric measurements (i.e., height, body weight, waist circumference, hip circumference), blood draw, and SAT biopsy. Body weight, body height, and blood pressure were assessed by trained personnel according to standard operating procedures [12,13]. Overall, six participants withdrew across the intervention phase. Two further participants had to be excluded from the second biopsy, one due to temporary use of anti-coagulants and the other one due to side effects (skin irritation) at the first biopsy (see Figure S1).

For the present project, the HELENA Trial cohort was categorized by quartiles of weight loss during the 12-week intervention phase, irrespective of the dietary method by which weight loss was achieved. These pooled analyses on the effects of overall weight loss on SAT gene expression were pre-specified in the study protocol [13] and were motivated by the fact that we had not observed significant differences between changes in gene expression by intermittent calorie restriction vs. continuous calorie restriction vs. control [12]. Since we aimed to investigate the effect of weight loss on SAT gene expression profiles, and because the number of participants with weight gain $(n=4$ with weight gain of $>2 \%$ ) was too small for well-powered analyses, we decided to exclude these participates. Thus, a sample of 138 participants was used for the present analyses.

\subsection{Blood-Based Biomarkers}

Blood draws from peripheral veins at the arm were conducted after a minimum of eight hours of overnight fasting. Routine metabolic biomarkers (fasting glucose, HDL cholesterol, LDL cholesterol, and total cholesterol) were quantified at the Central Laboratory of the University Hospital Heidelberg immediately after the blood draw, while fresh blood samples were processed, aliquoted, and stored at $-80^{\circ} \mathrm{C}$ at the Biobank of the National Center for Tumor Diseases (NCT, Heidelberg, Germany) in accordance with the regulations of the Biobank.

Serum concentrations of C-reactive protein (CRP) and insulin were measured on the Quickplex SQ 120 instrument from Meso Scale Discovery (MSD, Rockville, MD, USA) by electrochemiluminescence (ECL) using the manufacturer's proprietary kits. Hypoxia inducible lipid droplet-associated (HILPDA) and secreted frizzled-related protein 2 (SFRP2) were measured using enzyme-linked immunosorbent assays (ELISA; Emax Immunoassay System, Biozol, Eching, Germany). Intra-batch CVs were 9.1\% and $3.6 \%$ for HILPDA and SFRP2. Both ECL and ELISA quantifications were performed at the Division of Cancer Epidemiology laboratories, German Cancer Research Center (DKFZ) Heidelberg, Germany. Repeat samples from individual participants were positioned on the same analytical batch for biomarker quantification.

\subsection{Adipose Tissue Biopsies}

Details on the procedures for the local abdominal SAT biopsies have been described previously [12,13]. Briefly, SAT samples were obtained under local anesthesia by needle aspiration approximately 10-12 cm lateral to the umbilicus. All participants completed a minimum of eight hours of overnight fasting prior to sample collections. The SAT samples were immediately rinsed with sterile saline, snap-frozen in liquid nitrogen, aliquoted, and stored at $-80{ }^{\circ} \mathrm{C}$ at the Biobank of the National Center for Tumor Diseases (NCT, Heidelberg, Germany) for future analyses.

\section{4. $m R N A$ Extraction and Microarray Analyses}

Total mRNA extraction from SAT was done using the RNeasy Plus Universal Mini Kit (QIAGEN, Hilden, Germany) run on the QIAcube ${ }^{\circledR}$ (QIAGEN, Hilden, Germany), and the instructions of the manufacturer's protocol were followed. Integrity of RNA samples was measured on a Bioanalyzer 2100 (Agilent Technologies, Palo Alto, CA, USA). Samples with an mRNA Integrity Number above 
seven were stored at $-80{ }^{\circ} \mathrm{C}$ and used for microarray analyses with Human HT-12v4 Expression BeadChips (Illumina, San Diego, CA, USA), at the Genomics and Proteomics Core Facility of the German Cancer Research Center (DKFZ), Heidelberg.

The MicroArray data is available on ArrayExpress upon publication (accession number: E-MTAB-5926).

\subsection{Reverse Transcription and Quantitative Polymerase Chain Reaction (RT-qPCR)}

In accordance with the findings of our microarray experiment on differential gene expression by weight loss (see results section), three out of forty identified candidate genes (SCD, SFRP2, and HILPDA) were selected for RT-qPCR validation. This selection was made based on technical criteria, i.e., the feasibility to design primers, and scientific interest. For $S C D$, a central enzyme in fatty acid metabolism, it has previously been reported from smaller studies (see Table S1) that adipose tissue gene expression was downregulated with weight loss, which we intended to verify. Moreover, SCD may play a role in the development of cancer [15], which motivated our selection. In addition, we selected SFRP2 (implicated in WNT signaling, with a potential role in tumorigenesis [16]) and HILPDA (a potential regulator of lipid droplet formation and insulin sensitivity [17]) as more novel candidate genes. A validation of further genes was not possible due to the low amount of available fat tissue and due to financial restrictions.

Reverse transcription of mRNA was completed using SuperScript ${ }^{\mathrm{TM}}$ IV VILO ${ }^{\mathrm{TM}}$ (Invitrogen, ThermoFisher Scientific, Waltham, USA), while the total amount of mRNA was maintained across all reverse transcriptions. Validation of expression levels for selected candidate genes were performed by RT-qPCR (PikoReal ${ }^{\mathrm{TM}} 96$ Real Time PCR System, ThermoScientific ${ }^{\mathrm{TM}}$, Waltham, MA, USA) using the SYBR Green Master Mix (DyNAmo ColorFlash SYBR Green qPCR Kit, ThermoScientific, Waltham, MA, USA). The RT-qPCR settings with three technical replicates were implemented in accordance to the MIQE Guidelines (Minimum Information for Publication of Quantitative Real-Time PCR Experiments) [18,19]. Specific oligonucleotides sequences for RT-qPCR validation of stearoyl-CoA desaturase (SCD), SFRP2, and HILPDA were designed using Primer-BLAST, while high performance liquid chromatography purified primers were purchased from Eurofins Genomics (Eurofins Scientific SE, Luxemburg). Sequence accession numbers and primer sequences were as follows: HILPDA: NM_013332.3, forward $\left(5^{\prime} \rightarrow 3^{\prime}\right)$ : TGTTAGGTGTGGTACTGACCC and reverse $\left(5^{\prime} \rightarrow 3^{\prime}\right)$ : CTCTGTGTTGGCTAGTTGGC; SFRP2: NM_003013, forward $\left(5^{\prime} \rightarrow 3^{\prime}\right)$ : ATGCTTGAGTGCGACCGTTT and reverse $\left(5^{\prime} \rightarrow 3^{\prime}\right)$ : TACCTTTGGAGCTTCCTCGG; $S C D$ : NM_005063.4, forward $\left(5^{\prime} \rightarrow 3^{\prime}\right)$ : TCCAGAGGAGGTACTACAAACCT and reverse $\left(5^{\prime} \rightarrow 3^{\prime}\right)$ : GCACCACAGCATATCGCAAG. Expression levels of the target genes were normalized to the reference gene IPO8 (NM_006390.2, forward $\left(5^{\prime} \rightarrow 3^{\prime}\right)$ : CGAGCTCAACCAGTCCTACA and reverse $\left(5^{\prime} \rightarrow 3^{\prime}\right)$ : TCTGGCCAGTATTGTGTCACC) and analyzed with the delta-delta Ct method [20]. The criteria for selection of the reference gene were that IPO8 was not involved in energy metabolism, the expression level of IPO 8 was above the lower limit of detection in our microarray experiment, and the microarray data showed no changes in the expression levels between baseline and Week 12. Overall, specificity of the primers was insured by melt-cure analysis and 2D gel-electrophoresis. For two participants, mRNA sample volumes were limited so that the microarray gene expression results could not be validated by RT-qPCR.

\subsection{Literature Overview}

To provide an overview of previous studies on intentional weight loss induced by dietary interventions or bariatric-surgery and changes in the adipose tissue transcriptome (assessed by microarray analyses), we conducted a systematic literature search in PubMed. The identified studies are summarized in Table S1.

\subsection{Statistical Analyses}

Pre-processing of microarray data (log2 transformation, imputation of missings [21], and batch standardization by ComBat [22]) was conducted in Chipster 3.8. The analyses on gene expression 
profiles per weight loss categories were obtained on the basis of linear models with age and sex adjustment, using the limma package in $\mathrm{R}$ ( $\mathrm{R}$ Foundation for Statistical Computing) including Benjamini Hochberg correction for multiple testing. We did not further adjust for the initial study arm (intermittent calorie restriction vs. continuous calorie restriction vs. control), as the study arms were not associated with differences in SAT gene expression [12], and statistical adjustment thus only very marginally affected the statistical estimates. The same was true regarding BMI at baseline. Pathway analyses on weight loss-associated gene expression changes were done with parametric analyses of gene-set enrichment (PAGE), using the piano package in R including all significantly $(p<0.05)$ differentially regulated genes between lowest and highest weight loss quartile.

We conducted linear regression models in SAS 9.4 (Cary, NC, USA), adjusted for age and sex to analyze the overall effect of weight loss on $\log 2$ fold changes in both microarray- and RT-qPCR-derived expression levels of SFRP2, HILPDA, and SCD. In this context, p-values for pairwise comparison of least square means in gene expression between quartiles of weight loss (with the lowest weight loss quartile as the reference) were based on a t-test.

Linear mixed models for repeated measurement with age and sex adjustment were carried out in SAS 9.4 (Cary, NC, USA) to analyze time by treatment interaction effects on serum HILPDA and SFRP2 levels. The data on concentration levels are shown as mean values \pm SD (with normal $95 \%$ CIs) and relative changes over time as mean \pm SEM of log percentage changes.

Cross-sectional correlations between gene expression levels, BMI, and circulating biomarkers (HDL, LDL, insulin, HOMA-IR, CRP, SFRP2, HILPDA) were assessed by Spearman's coefficients adjusted for age and sex in $R$.

\section{Results}

\subsection{Characteristics of the Study Cohort}

The study group $(n=138)$ was categorized by weight loss as follows: Quartile 1 ( $n=34 ; \leq 2 \%$ weight gain to $\leq 1.9 \%$ weight loss, average: $0 \%$ ), Quartile 2 ( $n=35$; weight loss from $>1.9 \%$ to $\leq 4.5 \%$, average: $3.2 \%$ ), Quartile $3(n=34$, weight loss from $>4.5 \%$ to $\leq 7.5 \%$, average: $5.9 \%$ ), and Quartile $4(n=35$, weight loss $>7.5 \%$, average: $10.7 \%)$; participants in the four quartiles had similar baseline characteristics with regard to, age, sex, BMI, and routine metabolic biomarkers (see Table 1).

Table 1. Baseline characteristics of the study cohort by weight loss quartiles (138) ${ }^{1}$.

\begin{tabular}{lcccc}
\hline & Quartile 1 & Quartile 2 & Quartile 3 & Quartile 4 \\
\hline Women, $n$ (\%) & $(\boldsymbol{n}=\mathbf{3 5 )}$ & $\mathbf{( n = 3 4 )}$ & $\mathbf{( n = 3 5 )}$ & $\mathbf{( n = 3 4 )}$ \\
Age (years) & $17(48.6)$ & $18(52.9)$ & $14(40.0)$ & $18(52.9)$ \\
Weight (kg) & $47.2 \pm 8.3$ & $52.0 \pm 7.4$ & $50.6 \pm 8.7$ & $50.6 \pm 6.5$ \\
Height (cm) & $94.1 \pm 14.4$ & $93.2 \pm 15.9$ & $94.4 \pm 13.7$ & $94.5 \pm 16.3$ \\
BMI (kg/m $\left.{ }^{2}\right)$ & $173.4 \pm 8.0$ & $173.7 \pm 10.9$ & $173.6 \pm 9.4$ & $172.5 \pm 10.3$ \\
\hline Education level, $n(\%)$ & $31.5 \pm 3.7$ & $30.8 \pm 3.4$ & $31.3 \pm 3.8$ & $31.7 \pm 4.2$ \\
\hline Primary school & & & & \\
Secondary school & $4(12.1)$ & $2(5.9)$ & $5(14.7)$ & $2(5.7)$ \\
$\quad$ Higher education & $14(42.4)$ & $9(26.4)$ & $6(17.7)$ & $8(22.9)$ \\
\hline Glucose (mg/dL) & $15(45.5)$ & $23(67.7)$ & $23(67.6)$ & $25(71.4)$ \\
Insulin (mU/L) & $91.5 \pm 7.9$ & $95.1 \pm 6.6$ & $93.0 \pm 6.6$ & $93.0 \pm 7.8$ \\
HOMA-IR & $11.0 \pm 5.3$ & $10.6 \pm 5.0$ & $13.0 \pm 7.4$ & $13.6 \pm 7.0$ \\
Cholesterol (mg/dL) & $2.5 \pm 1.3$ & $2.5 \pm 1.2$ & $3.0 \pm 1.8$ & $3.1 \pm 1.6$ \\
HDL-cholesterol (mg/dL) & $212.8 \pm 35.2$ & $199.7 \pm 31.7$ & $214.9 \pm 36.8$ & $202.9 \pm 34.9$ \\
LDL-cholesterol (mg/dL) & $128.8 \pm 15.9$ & $51.8 \pm 13.5$ & $57.6 \pm 13.7$ & $52.9 \pm 15.1$ \\
\hline
\end{tabular}

${ }^{1}$ Data are shown as means \pm SD. ${ }^{2}$ Education level is missing for two participants. 


\subsection{Microarray Gene Expression by Weight Loss Groups}

There were 681 differentially expressed genes across weight loss groups ( $\left.p_{\text {adjusted }}<0.05\right)$. Out of these, 40 genes showed an absolute $\log _{2}$ FC of at least 0.4 between the lowest and the highest weight loss quartile (see Table 2). Fatty acid desaturase 1 (FADS1, FC = $1.09, p_{\text {adjusted }}<0.001$ ), SCD (FC $\left.=-1.00, p_{\text {adjusted }}<0.001\right)$, glycerol-3-phosphate acyltransferase (GPAM, FC = -0.54 , $\left.p_{\text {adjusted }}<0.001\right)$, and diazepam binding inhibitor $(D B I)\left(\mathrm{FC}=-0.44, p_{\text {adjusted }}<0.001\right)$, i.e., genes encoding key enzymes of lipid synthesis and cholesterol/fatty acid transport, were significantly downregulated among participants in the highest weight loss quartile. Likewise, several genes with functions in extracellular matrix modeling, cell-cell signaling, or cell differentiation were significantly downregulated (e.g., collagen, type XV, alpha 1 (COL15A1, FC $\left.=-0.50, p_{\text {adjusted }}<0.001\right)$, SFRP2 $\left(\mathrm{FC}=-0.65\right.$. $\left.p_{\text {adjusted }}=0.006\right)$, and HILPDA $\left.\left(\mathrm{FC}=-0.45, p_{\text {adjusted }}=0.001\right)\right)$. Highest fold changes among upregulated genes with weight loss were observed for complement component $6(C 6, \mathrm{FC}=0.66$, $\left.p_{\text {adjusted }}=0.001\right)$ and cell death-inducing DFFA-like effector A $\left(C I D E A, \mathrm{FC}=0.47, p_{\text {adjusted }}=0.015\right)$.

Parametric analyses of gene-set enrichment (PAGE), performed on microarray data for all 681 significantly differentially regulated genes $\left(p_{\text {adjusted }}<0.05\right)$, revealed a downregulation of lipid metabolism, cell membrane, and oxidation processes (e.g., GO:0006633_fatty acid biosynthetic process, GO:0035338_long-chain fatty-acyl-CoA biosynthetic process and GO:0006631_fatty acid metabolic process, GO:0005886_plasma membrane, and GO:0016491_oxidoreductase activity) (see Figure 1 and Table S2 for the top 50 enriched pathways).

A

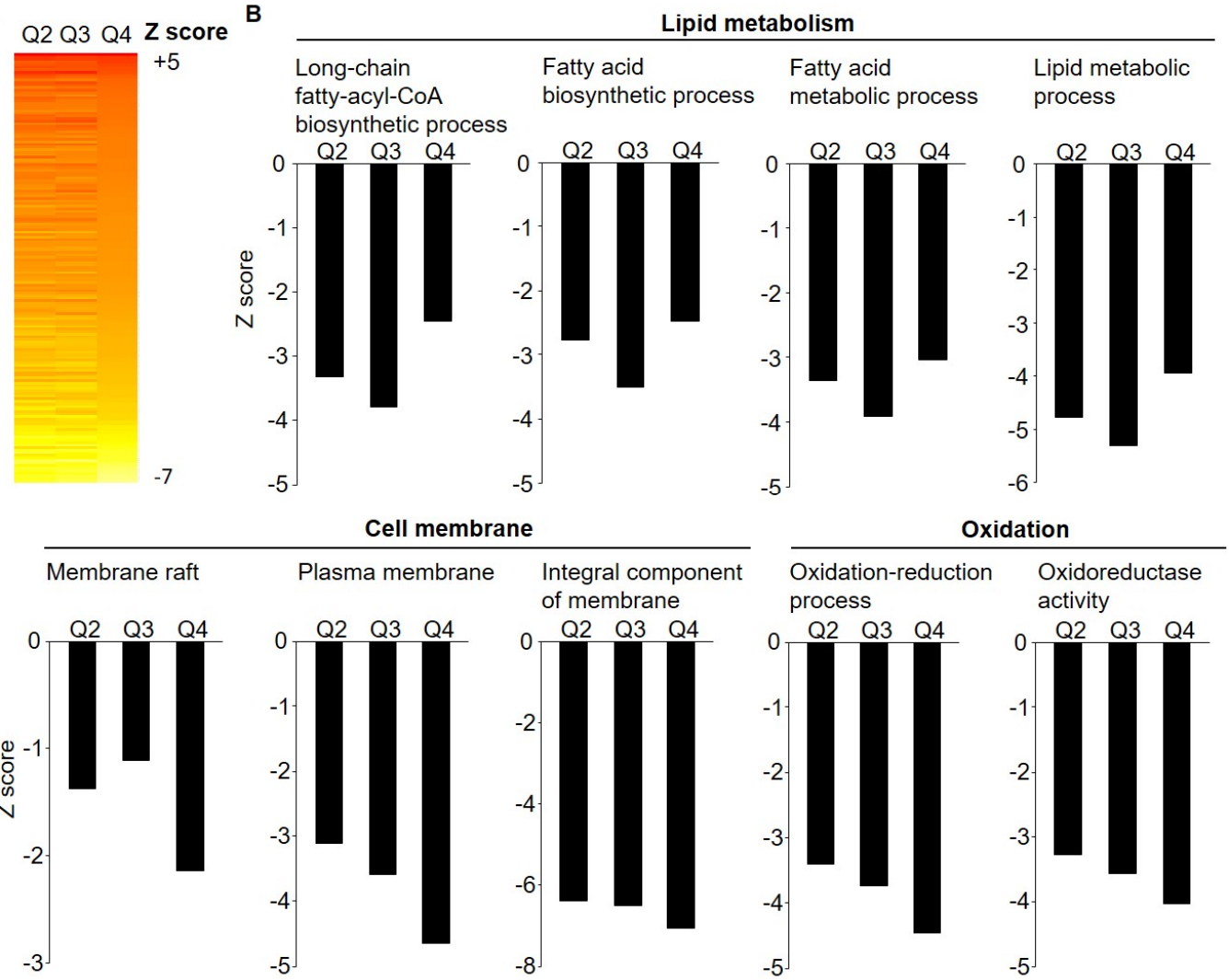

Figure 1. Effect of weight loss on SAT gene expression pathway enrichment $(n=138)$. Parametric analysis of gene-set enrichment (PAGE) were performed on microarray data including 681 significantly $(p<0.05)$ regulated genes across weight loss quartiles. (A) Heat map on biological pathways that were significantly differentially regulated by weight loss, based on the $\mathrm{Z}$ score for difference between weight loss quartiles with Q1 (lowest quartile) as a reference. (B) Biological pathways involved in lipid metabolism, cell membrane, and oxidation processes were significantly downregulated with weight loss. Further list of pathways see Table S2. 
Table 2. Up- and downregulated genes ( $p$-value $<0.05$; $\log _{2}$ fold change $>0.4$ ) for comparisons between highest- and lowest weight loss quartile $(n=138)$.

\begin{tabular}{|c|c|c|c|c|c|}
\hline Address & Gene & Description & Highest & Lowest & \\
\hline \multirow[t]{2}{*}{ ID } & \multirow[t]{2}{*}{ Symbol } & & \multicolumn{2}{|c|}{ Weight Loss Quartile } & \multirow[b]{2}{*}{$p_{\text {trend }}$ Value ${ }^{2}$} \\
\hline & & & $\log _{2} \mathrm{FC}^{1}$ & $p$ Value $^{1}$ & \\
\hline \multicolumn{6}{|c|}{ Downregulated genes } \\
\hline 2360020 & FADS1 & Fatty acid desaturase 1 & -1.09 & $<0.001$ & $<0.001$ \\
\hline 4850195 & SCD & Stearoyl-CoA desaturase & -1.00 & $<0.001$ & $<0.001$ \\
\hline 2140128 & SCD & Stearoyl-CoA desaturase & -0.69 & $<0.001$ & $<0.001$ \\
\hline 3060639 & SFRP2 & Secreted frizzled-related protein 2 & -0.65 & 0.006 & 0.004 \\
\hline 6200253 & THBS4 & Thrombospondin 4 & -0.58 & 0.019 & 0.026 \\
\hline 7040372 & GPAM & Glycerol-3-phosphate acyltransferase & -0.54 & $<0.001$ & 0.001 \\
\hline 7330544 & ALDOC & Aldolase $\mathrm{C}$, fructose-bisphosphate & -0.52 & $<0.001$ & 0.001 \\
\hline 3840026 & GPAM & Glycerol-3-phosphate acyltransferase & -0.51 & $<0.001$ & $<0.001$ \\
\hline 5090026 & COL15A1 & Collagen, type $\mathrm{XV}$, alpha 1 & -0.50 & $<0.001$ & $<0.001$ \\
\hline 5550292 & KLB & Klotho beta & -0.49 & $<0.001$ & $<0.001$ \\
\hline 240400 & PMEPA1 & Prostate transmembrane protein & -0.48 & 0.002 & 0.001 \\
\hline 4010709 & NNAT & Neuronatin & -0.47 & 0.008 & 0.005 \\
\hline 3390326 & ME1 & Malic enzyme 1, NADP(+)-dependent & -0.47 & $<0.001$ & $<0.001$ \\
\hline 5550379 & CAV1 & Caveolin 1 & -0.47 & 0.0041 & 0.017 \\
\hline 7650053 & ECHDC1 & Enoyl CoA hydratase domain containing 1 & -0.46 & $<0.001$ & $<0.001$ \\
\hline 450292 & TUBB2A & Tubulin & -0.46 & 0.008 & 0.011 \\
\hline 7320441 & HILPDA & Hypoxia inducible lipid droplet-associated & -0.45 & $<0.001$ & $<0.001$ \\
\hline 4480341 & DHCR24 & 24-dehydrocholesterol reductase & -0.45 & $<0.001$ & $<0.001$ \\
\hline 2370041 & LRRN3 & Leucine rich repeat neuronal 3 & -0.45 & $<0.001$ & 0.0012 \\
\hline 3450537 & DGAT2 & Diacylglycerol O-acyltransferase 2 & -0.44 & 0.015 & 0.016 \\
\hline 7610128 & KANK4 & KN motif and ankyrin repeat domains 4 & -0.44 & 0.032 & 0.012 \\
\hline 2480338 & DBI & Diazepam binding inhibitor & -0.44 & $<0.001$ & $<0.001$ \\
\hline 730040 & LAMB3 & Laminin, beta 3 & -0.43 & $<0.001$ & $<0.001$ \\
\hline 3830041 & PMEPA1 & Prostate transmembrane protein & -0.43 & 0.0061 & 0.0043 \\
\hline 360192 & INSIG1 & Insulin induced gene 1 & -0.43 & $<0.001$ & $<0.001$ \\
\hline 3180048 & IDH1 & Isocitrate dehydrogenase 1 & -0.42 & $<0.001$ & $<0.001$ \\
\hline 2230538 & LRRN3 & Leucine rich repeat neuronal 3 & -0.41 & 0.002 & 0.003 \\
\hline 1710484 & FMOD & Fibromodulin & -0.41 & 0.002 & 0.002 \\
\hline 3390343 & SREBF1 & Sterol regulatory element binding & -0.41 & 0.029 & 0.022 \\
\hline 3170594 & MAL2 & Mal, T-cell differentiation protein 2 & -0.41 & 0.013 & 0.020 \\
\hline 5310634 & FASN & Fatty acid synthase & -0.41 & 0.013 & 0.0118 \\
\hline 520474 & TENM4 & Teneurin transmembrane protein 4 & -0.40 & $<0.001$ & $<0.001$ \\
\hline \multicolumn{6}{|c|}{ Upregulated genes } \\
\hline 6520040 & C6 & Complement component 6 & 0.66 & $<0.001$ & $<0.001$ \\
\hline 6280370 & LOC646688 & Predicted: misc_RNA & 0.51 & $<0.001$ & $<0.001$ \\
\hline 2140278 & CIDEA & Cell death-inducing DFFA-like effector a & 0.47 & 0.015 & $<0.001$ \\
\hline 2490612 & ADH1B & Alcohol dehydrogenase IB & 0.45 & $<0.001$ & $<0.001$ \\
\hline 7560543 & MOCS1 & Molybdenum cofactor synthesis 1 & 0.44 & $<0.001$ & $<0.001$ \\
\hline 10048 & CIDEA & Cell death-inducing DFFA-like effector a & 0.44 & 0.012 & $<0.001$ \\
\hline 5490019 & GPX3 & Glutathione peroxidase 3 & 0.41 & 0.003 & $<0.001$ \\
\hline 6270372 & EGFLAM & $\begin{array}{l}\text { EGF-like, fibronectin type III and laminin G } \\
\text { domains }\end{array}$ & 0.40 & 0.012 & $<0.001$ \\
\hline
\end{tabular}

${ }^{1} p$ value (adjusted with Benjamini Hochberg) and $\log _{2}$ fold change (FC) calculations were based on a linear model from the limma package in $R$ for highest versus lowest weight loss quartile at Week $12 .{ }^{2} p_{\text {trend }}$ value (adjusted with Benjamini Hochberg) was based on linear model with weight as continuous covariate. 


\subsection{Validation of Gene Expression Levels for SCD, SFRP2, and HILPDA}

Consistent with the strong results for SCD in our microarray experiment, RT-qPCR validation revealed significant differences in SCD expression across weight loss groups $(p<0.001$; see Figure 2). The comparison of microarray and RT-qPCR data for SFRP2 and HILPDA shown in Figure 2 illustrates the overlapping results for differential expression with increasing weight loss with both quantification methods. Downregulation of these genes across weight loss groups followed a dose-response gradient.
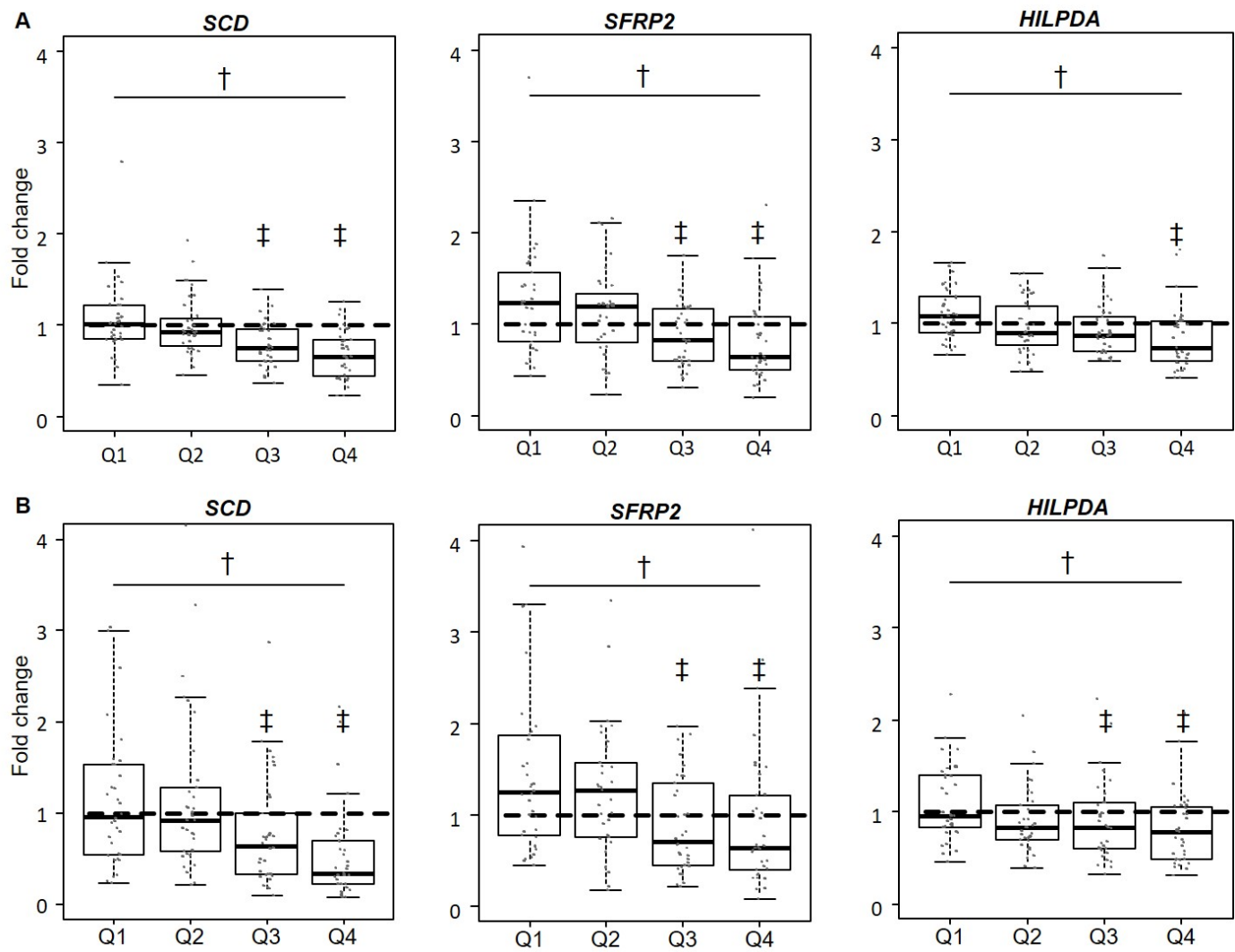

Figure 2. Effect of weight loss on SAT mRNA gene expression (microarray and RT-qPCR) of stearoyl-CoA desaturase (SCD), secreted frizzled-related protein 2 (SFRP2), and hypoxia inducible lipid droplet-associated protein (HILPDA). (A) Relative gene expression across weight loss quartiles (Q1: lowest quartile) determined by microarray $(n=138)$ is expressed as fold change $(F C)$ between baseline $\left(\mathrm{T}_{0}\right)$ and after 12 weeks $\left(\mathrm{T}_{1}\right)\left(\mathrm{FC}=2^{(\log \mathrm{T} 1-\log \mathrm{T} 0)}\right)$. (B) Expression profiles quantified by RT-qPCR $(n=$ 136) are presented as FC of the delta-delta CT method $\left(2^{-\Delta \Delta C T}\right)$. Data points with an FC $>4.2$ are not plotted (SCD: $n=2$, SFRP2: $n=1)$. Significant differences $(p<0.05)$ are depicted by $\dagger$ for overall effects and $\ddagger$ for pairwise comparisons.

\subsection{Circulating Levels of HILPDA and SFRP2}

Based on the results for downregulation of HILPDA and SFRP2 genes with weight loss, we selected these novel candidates to investigate if there are changes in circulating levels of the encoded proteins, again by weight loss quartiles. There were no significant differences $(p>0.05)$ between the weight loss quartiles for changes in circulating HILPDA or SFRP2 levels (see Table 3). Correlations between adipose tissue gene expression levels and corresponding serum protein concentrations of HILPDA and SFRP2 were low $(\rho<0.2)$ and not significant, both in cross-sectional analyses and with regard to changes in levels over time (see Figure 3 and Figure S2). 
Table 3. Change in serum concentrations of HILPDA and SFRP2 by weight loss quartiles ${ }^{1}$.

\begin{tabular}{|c|c|c|c|c|c|c|c|c|c|}
\hline & \multirow[t]{2}{*}{$n$} & \multirow{2}{*}{$\begin{array}{c}\text { Baseline } \\
\text { Mean } \pm \text { SD }\end{array}$} & \multirow[b]{2}{*}{$95 \%$ CI } & \multirow[t]{2}{*}{$n$} & \multicolumn{2}{|l|}{ Week 12} & \multirow{2}{*}{$\begin{array}{l}\text { Relative } \\
\text { Change }\end{array}$} & \multicolumn{2}{|c|}{$p$-Value ${ }^{1}$} \\
\hline & & & & & Mean \pm SD & $95 \%$ CI & & All & Q1 vs. Q4 \\
\hline \multicolumn{10}{|c|}{ HILPDA $(\mu \mathrm{g} / \mathrm{mL})$} \\
\hline Quartile 1 & 33 & $51.8 \pm 26.5$ & $(44.0,59.6)$ & 32 & $52.8 \pm 24.2$ & $(45.5,60.1)$ & $6.8 \pm 4.8$ & 0.78 & 0.84 \\
\hline Quartile 2 & 34 & $55.6 \pm 25.3$ & $(48.2,62.9)$ & 33 & $53.8 \pm 23.9$ & $(46.8,60.9)$ & $0.5 \pm 7.4$ & & \\
\hline Quartile 3 & 32 & $49.0 \pm 28.5$ & $(40.5,57.6)$ & 34 & $48.9 \pm 27.4$ & $(41.0,56.9)$ & $4.3 \pm 6.3$ & & \\
\hline Quartile 4 & 35 & $53.2 \pm 29.0$ & $(44.9,61.5)$ & 34 & $54.4 \pm 28.5$ & $(46.2,62.7)$ & $5.8 \pm 9.2$ & & \\
\hline \multicolumn{10}{|c|}{ SFRP2 (ng/mL) } \\
\hline Quartile 1 & 32 & $36.5 \pm 29.5$ & $(27.6,45.3)$ & 27 & $38.1 \pm 34.1$ & $(26.9,49.3)$ & $2.8 \pm 7.1$ & 0.36 & 0.31 \\
\hline Quartile 2 & 31 & $45.6 \pm 26.5$ & $(37.5,53.7)$ & 30 & $39.3 \pm 19.5$ & $(33.3,45.4)$ & $-3.0 \pm 5.3$ & & \\
\hline Quartile 3 & 31 & $29.3 \pm 13.6$ & $(25.2,33.4)$ & 32 & $33.2 \pm 15.3$ & $(28.7,37.8)$ & $11.2 \pm 8.9$ & & \\
\hline Quartile 4 & 33 & $45.0 \pm 27.5$ & $(36.9,53.1)$ & 31 & $46.1 \pm 26.1$ & $(38.2,54.0)$ & $-3.6 \pm 8.1$ & & \\
\hline
\end{tabular}

${ }^{1} p$ values for time by treatment interactions were calculated with linear mixed models adjusted for age and sex. Abbreviations: HILPDA, hypoxia inducible lipid droplet-associated protein; SFRP2, secreted frizzled-related protein 2.

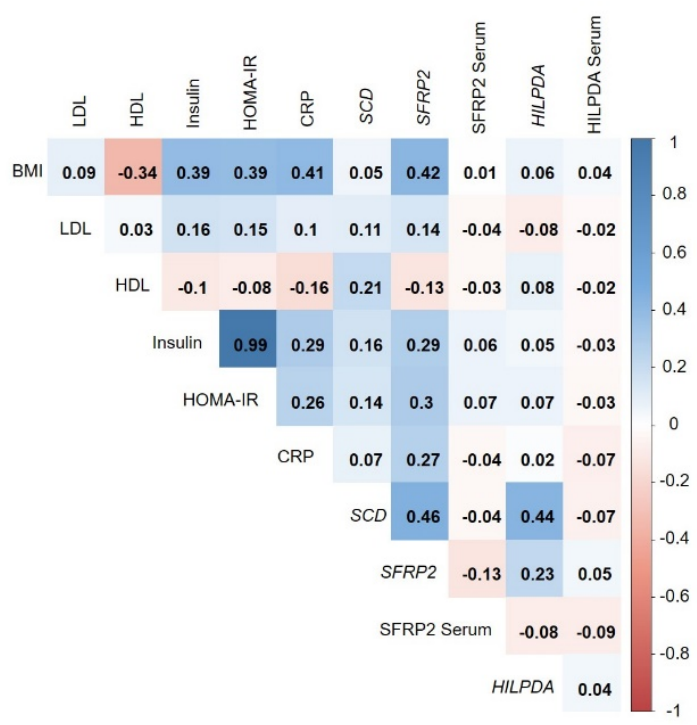

Figure 3. Cross-sectional correlations (at Week 12) between SCD, SFRP2, and HILPDA microarray gene expression levels, serum levels of SFRP2 and HILPDA, BMI, and metabolic biomarkers (LDL, HDL, insulin, HOMA-IR, CRP). Data are shown as Spearman coefficients adjusted for age and sex. The cross-sectional correlations for baseline data and for log relative changes between baseline and Week 12 are shown in Figure S2.

\subsection{Correlation of Adipose-Tissue Gene Expression with BMI and Metabolic Biomarkers}

Analyses on the correlates (BMI, HDL, LDL, insulin, HOMA-IR, and CRP) of gene expression levels of SFRP2, HILPDA, and SCD revealed a modest positive correlation between SFRP2 and BMI $(\rho=0.42$, see Figure 3). HILPDA and SCD showed no meaningful correlations $(\rho>0.4)$ with BMI or routine metabolic markers. There were no correlations between circulating SFRP2 and HILPDA levels and BMI or other metabolic markers. Results on cross-sectional analyses at Week 12 (Figure 3) were highly similar to those from baseline (Figure S2A). Analyses on changes in gene expression and biomarker levels over time showed only weak correlations at $\rho<0.4$ (Figure S2B). Correlations between expression levels of all 40 genes differentially regulated with weight loss in adipose tissue and BMI as well as established circulating markers of metabolism are shown in Figures S3-S5. Overall, correlations with BMI and classical disease risk biomarkers were weak. 


\section{Discussion}

In the present study we found that moderate weight loss induces differential expression of key enzymes of lipid synthesis and cholesterol transport as well as extracellular matrix remodeling in SAT. SCD, SFRP2, and HILPDA were identified and validated as novel adipose tissue biomarkers related to weight change. However, we observed no correlations between SAT gene expression levels of SFRP2 and HILPDA with blood levels of the proteins. Neither weight loss (prospectively) nor BMI (cross-sectionally) were related to circulating SFRP2 and HILPDA, suggesting that these adipose changes may not affect systemic blood levels. BMI showed a modest correlation with the expression of SFRP2, but not with the expression of SCD and HILPDA.

We observed pronounced effects of weight loss on the expression of SFRP2, a key modulator of WNT-signaling, in adipose tissue. Known functionality of SFRPs are modulations of growth factors belonging to the wingless-type mouse mammary tumor virus integration site (WNT) family. In general, WNT-signaling regulates cell differentiation and tissue remodeling in several tissue types including adipose tissue [23]. SFRP1, -2 and -4 bind to WNT-molecules and inhibit their actions with the consequence that adipogenesis is induced [24-26]. Against this background it is interesting to note that SFRP2 and -4 are significantly downregulated in SAT among gastrointestinal cancer patients with cachexia compared to weight stable patients [27]. Our finding on reduced SFRP2 expression with intentional weight loss supports the microarray-based result from two previous studies with approximately $10 \%$ weight loss, induced by low calorie diets [28,29]. Similarly, a significantly higher expression of SFRP2 and -3 in human SAT and VAT samples from obese individuals compared to non-obese has been reported, with more pronounced differences in VAT [30]. Expression levels for SFRP2 were positively correlated with insulin sensitivity (HOMA-IR; $r=0.49)$ and BMI $(r=0.65)$ in that study [30], while slightly weaker correlations were observed in the present project (HOMA-IR $r=0.28$ and BMI $r=0.42$ ). We did not observe a correlation for SFRP2 gene expression levels in SAT and circulating SFRP2. Moreover, unlike in one previous study [31] that showed significant positive associations between circulating SFRP2 and BMI as well as insulin levels, there were no such correlations in our study. Nevertheless, our data suggest that SFRP2 expression in adipose tissue can be controlled by moderate intentional weight loss. The implication of modifications in adipose tissue SFRP2 expression for chronic diseases such as cancer [16,32] requires further studies, however.

HILPDA is upregulated in both the VAT and SAT fraction of morbidly obese patients receiving bariatric surgery [17], which is in line with the present finding of decreased HILPDA expression in SAT with intentional CR-induced weight loss. There is one previous report indicating the same direction of change in HILPDA expression levels following a low-calorie diet for weight loss, although the FC magnitude was lower [29]. The highly conserved protein encoded by HILPDA is associated with lipid droplet formation especially in hepatocytes and adipocytes [33]. As a target of peroxisome proliferator activated receptor alpha (PPAR $\alpha)$, HILPDA might be involved in hepatic triglyceride turnover and be linked to insulin sensitivity [34]. Initially, HILPDA was identified in cervical cancer cells with activation under hypoxic conditions [35], which is why it was first termed hypoxia induced protein 2 (HIG2). Our results are particularly intriguing, because the functional involvement of HILPDA in cancer-related WNT-signaling and the potential use of this molecule as a novel therapeutic target for cancer treatment is under discussion [36,37].

The strongest changes in SAT gene expression with CR-induced weight loss in our study were observed for $S C D$ and FADS1, both rate limiting enzymes in the biosynthesis of unsaturated fatty acids. These results are in agreement with previous reports from $C R$ intervention and bariatric surgery trials $[6,7,28,29,38,39]$. Heightened activity of SCD, which catalyzes the synthesis of monounsaturated fatty acids, has been postulated to be a major checkpoint in the pathogenesis of obesity-driven diseases, in particular type 2 diabetes, cardiovascular diseases, and cancer [40,41]. FADS1 and FADS2 encode the proteins $\Delta 5$-desaturase and $\Delta 6$-desaturase which catalyze the conversion of linoleic acid and $\alpha$-linolenic acid into longer chained polyunsaturated fatty acids with more double bonds [42]. Large-scale prospective studies revealed that the activity levels of these enzymes are associated with 
the risk for type 2 diabetes, with the activity of $\Delta 5$-desaturase being inversely and the activity of $\Delta 6$-desaturase being directly related to disease risk [42]. In addition, there is evidence to suggest that altered FADS1 activity is at the interface between obesity and other major chronic diseases [43]. Future research is required to further unravel the metabolic consequences of decreased FADS1 gene expression in adipose tissue with weight loss.

There were several additional genes among our top hits that confirm findings from previous transcriptomics analyses of adipose tissue, which we identified by a systematic literature search on microarray-based trials for differential adipose tissue gene expression after CR- and bariatric surgery-induced weight loss (overlaps to our study are shown in Table S1). Interestingly, a recently published analysis from the DIOGENES trial including 191 obese participants undergoing a low-calorie diet for 8 weeks with $\sim 10 \%$ weight loss, revealed 350 genes that were differentially regulated after weight loss (absolute FC $\geq 0.2$ and corrected $p$-values < 0.05) [29]. In this study, 37 genes showed FCs with weight loss at a magnitude $\geq 0.4$ (the fold change cut-point in our study) [29], out of which $S C D$, SFRP2, THBS4, and GPAM were among the top hits in our study as well (i.e., FC $\geq 0.4$ ) [29].

The main strength of this study was the size of the study cohort, which was entirely characterized by microarray-based gene expression profiling, with additional validation of the top hits by RT-qPCR. Nevertheless, it must be acknowledged that we could not use VAT samples for gene expression analyses, as taking VAT biopsies was not possible for ethical and practical reasons. It was neither feasible to separate the tissue biopsy into different cellular fractions prior to mRNA extraction. It also should be noted that our trial comprised a rather homogeneous group of overweight and obese individuals without metabolic co-morbidities. Thus, we cannot generalize our results to other study populations (e.g., diabetics, morbidly obese individuals, or individuals undergoing stronger weight fluctuations). Unlike previous studies, in which SAT gene expression experiments facilitated the identification of circulating adipokines [44], we did not observe associations between SAT gene expression levels and blood levels of SFRP2 and HILPDA. We did not have the opportunity to expand our work to laboratory-based mechanistic analyses, and functional aspects of our findings from post hoc analyses of RCT data need to be investigated by controlled wet lab studies before firm conclusions on biological mechanisms can be drawn. Finally, the number of genes that we could validate was restricted due to financial reasons and limited adipose tissue sample availability.

\section{Conclusions}

In summary, our study showed differential expression of genes in subcutaneous adipose tissue encoding proteins, which may reduce lipid biosynthesis, facilitate lipid utilization, alter WNT-signaling, and convert adipose tissue structure with weight loss. Mechanistic studies are needed to investigate whether proteins such as SFRP2, HILPDA, and SCD mediate associations between obesity and its comorbidities.

Supplementary Materials: The following are available online at http:/ / www.mdpi.com/2072-6643/11/3/639/ s1. Figure S1: Participation rates for the intervention phase of the HELENA Trial; Figure S2: Cross-sectional correlations between SCD, SFRP2, and HILPDA microarray gene expression levels, serum levels of SFRP2 and HILPDA, BMI, and metabolic biomarkers (LDL-cholesterol, HDL cholesterol, insulin, HOMA-IR, CRP); Figure S3: Cross-sectional correlations between microarray gene expression levels, BMI, and metabolic biomarkers (LDL-cholesterol, HDL-cholesterol, insulin, HOMA-IR, CRP) at baseline; Figure S4: Cross-sectional correlations between microarray gene expression levels, BMI, and metabolic biomarkers (LDL-cholesterol, HDL-cholesterol, insulin, HOMA-IR, CRP) at Week 12; Figure S5: Cross-sectional correlations between microarray gene expression levels, BMI, and metabolic biomarkers (LDL-cholesterol, HDL-cholesterol, insulin, HOMA-IR, CRP) for log relative changes between baseline and Week 12; Table S1: Literature review on diet-(SAT samples) and bariatric surgery-(SAT and VAT samples) induced weight loss with microarray gene expression of adipose tissue; Table S2: Top 50 enriched pathways between lowest (reference) and highest weight loss quartile.

Author Contributions: Conceptualization, R.S., C.M.U., M.K., R.K., and T.K.; methodology, R.S., D.S., and J.G.; validation, R.S. and J.G.; formal analysis, D.S., R.S., and J.G.; investigation, R.S., M.E.G., and T.K.; resources, R.S., T.S.J., C.M.U., R.K., and T.K.; data curation, R.S. and D.S.; writing-original draft preparation, R.S. and T.K.; writing-review and editing, all authors; visualization, R.S., J.G., and D.S.; supervision, T.K.; project administration, R.S.; funding acquisition, C.M.U., R.K., T.K., and R.S. 
Funding: The Trial was registered at ClinicalTrials.gov (NCT02449148) prior to enrollment and was funded by the Helmholtz Association of German Research Centers (Cross Program Topic: Metabolic Dysfunction). Ulrich was funded by the Huntsman Cancer Foundation, Salt Lake City, Utah, USA, and NIH U01 CA 206110.

Acknowledgments: The authors thank all HELENA Trial participants and our study team. Biomaterial samples were processed by the Biobank of the National Center for Tumor Diseases (NCT, Heidelberg, Germany) in accordance with the regulations of the Biobank. We also thank the microarray unit of the DKFZ Genomics and Proteomics Core Facility for providing the Illumina Whole Genome Expression BeadChips and related services.

Conflicts of Interest: The authors declare no conflict of interest. The funders had no role in the design of the study; in the collection, analyses, or interpretation of data; in the writing of the manuscript; or in the decision to publish the results.

\section{References}

1. Renehan, A.G.; Zwahlen, M.; Egger, M. Adiposity and cancer risk: New mechanistic insights from epidemiology. Nat. Rev. Cancer 2015, 15, 484-498. [CrossRef]

2. Calle, E.E.; Kaaks, R. Overweight, obesity and cancer: Epidemiological evidence and proposed mechanisms. Nat. Rev. Cancer 2004, 4, 579-591. [CrossRef]

3. Tchernof, A.; Despres, J.P. Pathophysiology of human visceral obesity: An update. Physiol. Rev. 2013, 93, 359-404. [CrossRef]

4. Tilg, H.; Moschen, A.R. Adipocytokines: Mediators linking adipose tissue, inflammation and immunity. Nat. Rev. Immunol. 2006, 6, 772-783. [CrossRef]

5. Jensen, M.D.; Ryan, D.H.; Apovian, C.M.; Ard, J.D.; Comuzzie, A.G.; Donato, K.A.; Hu, F.B.; Hubbard, V.S.; Jakicic, J.M.; Kushner, R.F.; et al. 2013 AHA/ACC/TOS guideline for the management of overweight and obesity in adults. Circulation 2013, 129, S102-S138. [CrossRef]

6. Magkos, F.; Fraterrigo, G.; Yoshino, J.; Luecking, C.; Kirbach, K.; Kelly, S.C.; de Las Fuentes, L.; He, S.; Okunade, A.L.; Patterson, B.W.; et al. Effects of moderate and subsequent progressive weight loss on metabolic function and adipose tissue biology in humans with obesity. Cell Metab. 2016, 23, 591-601. [CrossRef]

7. Mutch, D.M.; Pers, T.H.; Temanni, M.R.; Pelloux, V.; Marquez-Quinones, A.; Holst, C.; Martinez, J.A.; Babalis, D.; van Baak, M.A.; Handjieva-Darlenska, T.; et al. A distinct adipose tissue gene expression response to caloric restriction predicts 6-mo weight maintenance in obese subjects. Am. J. Clin. Nutr. 2011, 94, 1399-1409. [CrossRef]

8. Klimcakova, E.; Roussel, B.; Marquez-Quinones, A.; Kovacova, Z.; Kovacikova, M.; Combes, M.; Siklova-Vitkova, M.; Hejnova, J.; Sramkova, P.; Bouloumie, A.; et al. Worsening of obesity and metabolic status yields similar molecular adaptations in human subcutaneous and visceral adipose tissue: Decreased metabolism and increased immune response. J. Clin. Endocrinol. Metab. 2011, 96, E73-E82. [CrossRef]

9. Moreno-Navarrete, J.M.; Petrov, P.; Serrano, M.; Ortega, F.; Garcia-Ruiz, E.; Oliver, P.; Ribot, J.; Ricart, W.; Palou, A.; Bonet, M.L.; et al. Decreased rb1 mrna, protein, and activity reflect obesity-induced altered adipogenic capacity in human adipose tissue. Diabetes 2013, 62, 1923-1931. [CrossRef]

10. Mardinoglu, A.; Heiker, J.T.; Gartner, D.; Bjornson, E.; Schon, M.R.; Flehmig, G.; Kloting, N.; Krohn, K.; Fasshauer, M.; Stumvoll, M.; et al. Extensive weight loss reveals distinct gene expression changes in human subcutaneous and visceral adipose tissue. Sci. Rep. 2015, 5, 14841. [CrossRef]

11. Liu, Y.; Aron-Wisnewsky, J.; Marcelin, G.; Genser, L.; Le Naour, G.; Torcivia, A.; Bauvois, B.; Bouchet, S.; Pelloux, V.; Sasso, M.; et al. Accumulation and changes in composition of collagens in subcutaneous adipose tissue after bariatric surgery. J. Clin. Endocrinol. Metab. 2016, 101, 293-304. [CrossRef]

12. Schübel, R.; Nattenmüller, J.; Sookthai, D.; Nonnenmacher, T.; Graf, M.E.; Riedl, L.; Schlett, C.L.; von Stackelberg, O.; Johnson, T.; Nabers, D.; et al. Effects of intermittent and continuous calorie restriction on body weight and metabolism over 50 weeks: A randomized controlled trial. Am. J. Clin. Nutr. 2018, 108, 933-945. [CrossRef]

13. Schübel, R.; Graf, M.E.; Nattenmüller, J.; Nabers, D.; Sookthai, D.; Gruner, L.F.; Johnson, T.; Schlett, C.L.; von Stackelberg, O.; Kirsten, R.; et al. The effects of intermittent calorie restriction on metabolic health: Rationale and study design of the helena trial. Contemp. Clin. Trials 2016, 51, 28-33. [CrossRef] 
14. Oberritter, H.; Schabethal, K.; von Ruesten, A.; Boeing, H. The dge nutrition circle-Presentation and basis of the food-related recommendations from the german nutrition society (dge). Ernahrungs Umschau 2013, 60, 24-29.

15. Pakiet, A.; Kobiela, J.; Stepnowski, P.; Sledzinski, T.; Mika, A. Changes in lipids composition and metabolism in colorectal cancer: A review. Lipids Health Dis. 2019, 18, 29. [CrossRef]

16. Liu, Y.; Zhou, Q.; Zhou, D.; Huang, C.; Meng, X.; Li, J. Secreted frizzled-related protein 2-mediated cancer events: Friend or foe? Pharmacol. Rep. 2017, 69, 403-408. [CrossRef]

17. DiStefano, M.T.; Roth Flach, R.J.; Senol-Cosar, O.; Danai, L.V.; Virbasius, J.V.; Nicoloro, S.M.; Straubhaar, J.; Dagdeviren, S.; Wabitsch, M.; Gupta, O.T.; et al. Adipocyte-specific hypoxia-inducible gene 2 promotes fat deposition and diet-induced insulin resistance. Mol. Metab. 2016, 5, 1149-1161. [CrossRef]

18. Bustin, S.A.; Benes, V.; Garson, J.A.; Hellemans, J.; Huggett, J.; Kubista, M.; Mueller, R.; Nolan, T.; Pfaffl, M.W.; Shipley, G.L.; et al. The miqe guidelines: Minimum information for publication of quantitative real-time pcr experiments. Clin. Chem. 2009, 55, 611-622. [CrossRef] [PubMed]

19. Taylor, S.; Wakem, M.; Dijkman, G.; Alsarraj, M.; Nguyen, M. A practical approach to rt-qpcr-publishing data that conform to the miqe guidelines. Methods 2010, 50, S1-S5. [CrossRef]

20. Livak, K.J.; Schmittgen, T.D. Analysis of relative gene expression data using real-time quantitative pcr and the 2(-delta delta c(t)) method. Methods 2001, 25, 402-408. [CrossRef]

21. Troyanskaya, O.; Cantor, M.; Sherlock, G.; Brown, P.; Hastie, T.; Tibshirani, R.; Botstein, D.; Altman, R.B. Missing value estimation methods for DNA microarrays. Bioinformatics 2001, 17, 520-525. [CrossRef] [PubMed]

22. Johnson, W.E.; Li, C.; Rabinovic, A. Adjusting batch effects in microarray expression data using empirical bayes methods. Biostatistics 2007, 8, 118-127. [CrossRef] [PubMed]

23. Christodoulides, C.; Lagathu, C.; Sethi, J.K.; Vidal-Puig, A. Adipogenesis and wnt signalling. Trends Endocrinol. Metab. 2009, 20, 16-24. [CrossRef]

24. Park, J.R.; Jung, J.W.; Lee, Y.S.; Kang, K.S. The roles of wnt antagonists dkk1 and sfrp4 during adipogenesis of human adipose tissue-derived mesenchymal stem cells. Cell Prolif. 2008, 41, 859-874. [CrossRef] [PubMed]

25. Lagathu, C.; Christodoulides, C.; Tan, C.Y.; Virtue, S.; Laudes, M.; Campbell, M.; Ishikawa, K.; Ortega, F.; Tinahones, F.J.; Fernandez-Real, J.M.; et al. Secreted frizzled-related protein 1 regulates adipose tissue expansion and is dysregulated in severe obesity. Int. J. Obes. 2010, 34, 1695-1705. [CrossRef] [PubMed]

26. Cianferotti, L.; Demay, M.B. Vdr-mediated inhibition of dkk1 and sfrp2 suppresses adipogenic differentiation of murine bone marrow stromal cells. J. Cell. Biochem. 2007, 101, 80-88. [CrossRef] [PubMed]

27. Dahlman, I.; Mejhert, N.; Linder, K.; Agustsson, T.; Mutch, D.M.; Kulyte, A.; Isaksson, B.; Permert, J.; Petrovic, N.; Nedergaard, J.; et al. Adipose tissue pathways involved in weight loss of cancer cachexia. Br. J. Cancer 2010, 102, 1541-1548. [CrossRef] [PubMed]

28. Johansson, L.E.; Danielsson, A.P.; Parikh, H.; Klintenberg, M.; Norstrom, F.; Groop, L.; Ridderstrale, M. Differential gene expression in adipose tissue from obese human subjects during weight loss and weight maintenance. Am. J. Clin. Nutr. 2012, 96, 196-207. [CrossRef]

29. Armenise, C.; Lefebvre, G.; Carayol, J.; Bonnel, S.; Bolton, J.; Di Cara, A.; Gheldof, N.; Descombes, P.; Langin, D.; Saris, W.H.; et al. Transcriptome profiling from adipose tissue during a low-calorie diet reveals predictors of weight and glycemic outcomes in obese, nondiabetic subjects. Am. J. Clin. Nutr. 2017, 106, 736-746. [CrossRef]

30. Ehrlund, A.; Mejhert, N.; Lorente-Cebrian, S.; Astrom, G.; Dahlman, I.; Laurencikiene, J.; Ryden, M. Characterization of the wnt inhibitors secreted frizzled-related proteins (sfrps) in human adipose tissue. J. Clin. Endocrinol. Metab. 2013, 98, E503-E508. [CrossRef]

31. Crowley, R.K.; O’Reilly, M.W.; Bujalska, I.J.; Hassan-Smith, Z.K.; Hazlehurst, J.M.; Foucault, D.R.; Stewart, P.M.; Tomlinson, J.W. Sfrp2 is associated with increased adiposity and vegf expression. PLoS ONE 2016, 11, e0163777. [CrossRef] [PubMed]

32. Kaur, A.; Webster, M.R.; Marchbank, K.; Behera, R.; Ndoye, A.; Kugel, C.H., 3rd; Dang, V.M.; Appleton, J.; O'Connell, M.P.; Cheng, P.; et al. Sfrp2 in the aged microenvironment drives melanoma metastasis and therapy resistance. Nature 2016, 532, 250-254. [CrossRef] [PubMed]

33. DiStefano, M.T.; Danai, L.V.; Roth Flach, R.J.; Chawla, A.; Pedersen, D.J.; Guilherme, A.; Czech, M.P. The lipid droplet protein hypoxia-inducible gene 2 promotes hepatic triglyceride deposition by inhibiting lipolysis. J. Biol. Chem. 2015, 290, 15175-15184. [CrossRef] [PubMed] 
34. Mattijssen, F.; Georgiadi, A.; Andasarie, T.; Szalowska, E.; Zota, A.; Krones-Herzig, A.; Heier, C.; Ratman, D.; De Bosscher, K.; Qi, L.; et al. Hypoxia-inducible lipid droplet-associated (hilpda) is a novel peroxisome proliferator-activated receptor (ppar) target involved in hepatic triglyceride secretion. J. Biol. Chem. 2014, 289, 19279-19293. [CrossRef] [PubMed]

35. Denko, N.; Schindler, C.; Koong, A.; Laderoute, K.; Green, C.; Giaccia, A. Epigenetic regulation of gene expression in cervical cancer cells by the tumor microenvironment. Clin. Cancer Res. 2000, 6, 480-487.

36. Gimm, T.; Wiese, M.; Teschemacher, B.; Deggerich, A.; Schodel, J.; Knaup, K.X.; Hackenbeck, T.; Hellerbrand, C.; Amann, K.; Wiesener, M.S.; et al. Hypoxia-inducible protein 2 is a novel lipid droplet protein and a specific target gene of hypoxia-inducible factor-1. FASEB J. 2010, 24, 4443-4458. [CrossRef]

37. Applebaum, M.A.; Jha, A.R.; Kao, C.; Hernandez, K.M.; DeWane, G.; Salwen, H.R.; Chlenski, A.; Dobratic, M.; Mariani, C.J.; Godley, L.A.; et al. Integrative genomics reveals hypoxia inducible genes that are associated with a poor prognosis in neuroblastoma patients. Oncotarget 2016, 7, 76816-76826. [CrossRef]

38. Franck, N.; Gummesson, A.; Jernas, M.; Glad, C.; Svensson, P.A.; Guillot, G.; Rudemo, M.; Nystrom, F.H.; Carlsson, L.M.; Olsson, B. Identification of adipocyte genes regulated by caloric intake. J. Clin. Endocrinol. Metab. 2011, 96, E413-E418. [CrossRef]

39. Capel, F.; Viguerie, N.; Vega, N.; Dejean, S.; Arner, P.; Klimcakova, E.; Martinez, J.A.; Saris, W.H.; Holst, C.; Taylor, M.; et al. Contribution of energy restriction and macronutrient composition to changes in adipose tissue gene expression during dietary weight-loss programs in obese women. J. Clin. Endocrinol. Metab. 2008, 93, 4315-4322. [CrossRef]

40. Poudyal, H.; Brown, L. Stearoyl-coa desaturase: A vital checkpoint in the development and progression of obesity. Endocr. Metab. Immune Disorders Drug Targets 2011, 11, 217-231. [CrossRef]

41. Ntambi, J.M.; Miyazaki, M. Regulation of stearoyl-coa desaturases and role in metabolism. Prog. Lipid Res. 2004, 43, 91-104. [CrossRef]

42. Kroger, J.; Schulze, M.B. Recent insights into the relation of delta5 desaturase and delta6 desaturase activity to the development of type 2 diabetes. Curr. Opin. Lipidol. 2012, 23, 4-10. [CrossRef] [PubMed]

43. Tosi, F.; Sartori, F.; Guarini, P.; Olivieri, O.; Martinelli, N. Delta-5 and delta-6 desaturases: Crucial enzymes in polyunsaturated fatty acid-related pathways with pleiotropic influences in health and disease. Adv. Exp. Med. Biol. 2014, 824, 61-81.

44. Inadera, $\mathrm{H}$. The usefulness of circulating adipokine levels for the assessment of obesity-related health problems. Int. J. Med. Sci. 2008, 5, 248-262. [CrossRef] 